This is the peer reviewed version of the following article: Fenni, SE, Wang, J, Haddaoui, N, Favis, BD, Müller, AJ, Cavallo, D. Nucleation modalities in poly(lactide), poly(butylene succinate), and poly(E-caprolactone) ternary blends with partial wetting morphology. Polymer Crystallization. 2020; 3:e10145. , which has been published in final form at https://doi.org/10.1002/pcr2.10145. This article may be used for non-commercial purposes in accordance with Wiley Terms and Conditions for Use of Self-Archived Versions

\title{
Nucleation modalities in poly(lactide), poly(butylene succinate) and poly( $\varepsilon$-caprolactone) ternary blends with partial wetting morphology
}

Seif Eddine Fenni ${ }^{1,2}$, Jun Wang ${ }^{3}$, Nacerddine Haddaoui ${ }^{2}$, Basil D. Favis ${ }^{3 *}$, Alejandro J. Müller ${ }^{4,5^{*}}$, and Dario Cavallo ${ }^{1^{*}}$

1. Department of Chemistry and Industrial Chemistry, University of Genova, via Dodecaneso, 31 - 16146 Genova, Italy

2. Laboratory of Physical-Chemistry of High Polymers (LPCHP), Faculty of Technology, University of Ferhat ABBAS Sétif-1, 19000 Sétif, Algeria

3. CREPEC, Department of Chemical Engineering, École Polytechnique de Montréal, Montréal, Québec H3C3A7, Canada

4. POLYMAT and Polymer Science and Technology Department, Faculty of Chemistry, University of the Basque Country UPV/EHU, Paseo Manuel de Lardizábal 3, 20018 Donostia-San Sebastián, Spain 5. IKERBASQUE, Basque Foundation for Science, Bilbao, Spain

Corresponding authors:

basil.favis@polymtl.ca; alejandrojesus.muller@ehu.es; dario.cavallo@unige.it 


\begin{abstract}
Ternary biodegradable polymer blends of poly (lactic acid) (PLA), poly (butylene succinate) (PBS), and poly ( $\varepsilon$-caprolactone) (PCL) with the composition 45/10/45 wt\% and exhibiting partial-wetting morphology were prepared. In this morphology, the minor phase is present as self-assembled droplets at the co-continuous interface of the other two major phases. The crystallization of the components in the various blends was thoroughly investigated. Differential scanning calorimetry highlighted minor differences in the overall kinetics of a given component in the ternary blend, with respect to the neat polymer. On the other hand, several unusual nucleation mechanisms could be studied by polarized optical microscopy (PLOM). With reference to the major phases, PLA spherulites displayed surfaceinduced nucleation from the interface with molten PBS or PCL droplets. On lowering the crystallization temperature, the PBS phase effectively nucleated at the interface with previously crystallized PLA domains, forming a transcrystalline morphology. Concerning the minor phase, weak partial-wetting PBS droplets displayed a droplet-to-droplet percolation of the nucleation events. Strongly partial-wetting PCL droplets were confined between previously crystallized PLA and PBS co-continuous phases and, instead, solidified as isolated domains randomly in space. This work provides further insights in the relationship between morphology and crystallization in immiscible ternary blends.
\end{abstract}

\title{
1. Introduction
}

Recently, significant attention has been given to biodegradable and biobased polymers in order to replace petroleum-based materials and hence reduce their environmental footprint [1-2]. Among other biodegradable polymers, Poly (lactic acid) (PLA), poly (butylene succinate) (PBS), poly ( $\varepsilon$-caprolactone) (PCL), and their immiscible blends have been largely studied ${ }^{[3-6]}$. Polymer blending can significantly influence the crystallization behavior in which the nucleation step and kinetic aspects are, in most cases, substantially different from those of the pure blend components.

Depending on the morphology of the prepared immiscible blend, the crystallization kinetics can differ largely. For instance, in blends with a sea-island morphology it can be first order for the minor phase, or sigmoidal for the major phase. In blends exhibiting the cocontinuous morphology, the most encountered kinetics for both blend components is sigmoidal kinetics, due to the percolated structure which allows a given nucleation event to spread unhindered in the domain ${ }^{[7]}$. 
In the crystallization of immiscible blends, with respect to the growth stage, it is the nucleation step which is the most significantly affected. Thus, peculiar nucleation events can arise. For a given component, the nucleation can be enhanced leading to a faster overall crystallization kinetics. This enhancement can be mostly related to interface-induced nucleation due to the presence of the contact surface with the other component, or to some impurities transfer from the other polymer during melt processing. On the other hand, the nucleation rate can also be reduced when the polymer is present as a minor component. This last effect is explained by the lack of active heterogeneities (in the minor component) responsible for nucleation in the bulk polymer, so that the overall crystallization process becomes fractionated ${ }^{[8-11]}$. Recently Fenni et al. [12] have reviewed the nucleation and crystallization of biopolymers (including PLA, PCL, PBS, and PHB) in their immiscible blends.

Interface-induced (or interface-assisted) nucleation has been previously reported in blends of crystalline/crystalline or crystalline/amorphous components ${ }^{[12-18]}$. The nucleation phenomenon can be easily visualized by polarized optical microscopy (PLOM) and it has been attributed to different mechanisms such as: the existence of an epitaxial relationship between the structures of the crystalline components ${ }^{[12]}$; reduction of the energy barrier needed for nuclei formation due to phase separation [19-23]; shear and "fluctuation-induced" nucleation, which promote some local chain orientation at the interface ${ }^{[15]}$; chemical and/or physical interaction between the functional groups of polymer chains at the interface during melt processing ${ }^{[22,24]}$; and local miscibility between the phase components ${ }^{[13,25-28]}$. On the other hand, some studies attributed the observed surface-induced nucleation to the migration of impurities and heterogeneities to the interface between phases during the mixing stage ${ }^{[19]}$.

Recently, multicomponent immiscible blends (blends with more than two components) have attracted considerable attention due to their mechanical performance enhancement and other functional application potential ${ }^{[29-33]}$. Two main types of morphology can be obtained in multiphase polymer blends with three components (e.g., immiscible ternary blends with two major components and one minor phase) which are complete-wetting and partial-wetting. In the latter, the minor component is present as droplets self-assembled at the interface of the other two major components ${ }^{[34-39]}$. The mechanism behind the produced morphology is the result of the spreading coefficients which express the interfacial tension and interfacial force equilibrium between different blend components [12,40-42]. The partial wetting of ternary polymer mixtures has not been extensively studied and remains a new frontier. 
A partial wetting model system in this respect is constituted by the ternary blends based on PLA, PCL, and PBS. In fact, for the characteristic spreading coefficients, when any of the polymers is present as the minor component (for example in the range of 10 volume percent or less), it can form partially-wet droplets at the interface of the other two major components. Recently, we have investigated in detail the crystallization behavior of PLA partially-wet droplets in contact with molten PCL and PBS phases ${ }^{[43]}$. It was found that the nucleation of PLA spherulites can occur at the interface with molten polymers, with a meaningful preference for PCL, which was explained by taking into account nucleation theory and interfacial tension differences among the PLA/PCL and PLA/PBS polymer pairs. Furthermore, the percolation of crystallization domains between adjacent PLA droplets was found to be possible, due to the weak partial wetting regime of this system, which enabled a transition to thin completely-wet regions in the melt, followed by a slow de-wetting during crystallization ${ }^{[43]}$.

This work explores the relation between crystallization kinetics/nucleation and blend morphology, for PLA/PCL/PBS ternary blends exhibiting a partial-wetting state. In particular, the overall crystallization kinetics of all the phases is investigated via differential scanning calorimetry (DSC), while the morphology and nucleation modalities of both major PBS and PLA phases and minor PBS and PCL components are studied via PLOM. The results obtained from partially wet PBS and PCL droplets are compared with the previous work on PLA droplets ${ }^{[43]}$. In fact, PBS droplets provide the case where crystallization occurs while in contact with one solid and one molten phase (PLA and PCL, respectively) and PCL droplets solidify while confined by two solid semicrystalline major phases (PLA and PBS).

\section{Materials and Experiments}

\subsection{Materials}

Poly lactic acid (PLA) (Ingeo 3001D) was purchased from NatureWorks. It has a weight average molar mass equal to $155 \mathrm{~kg} / \mathrm{mol}$ and D-isomer content of about $1.4 \%$. Poly(butylene succinate) (PBS) (1001MD) with a weight average molar mass of $60 \mathrm{~kg} / \mathrm{mol}$ was purchased from Showa Denko. Polycaprolactone (PCL) (Capa ${ }^{\mathrm{TM}}$ 6800) was purchased from Perstorp. The weight average molar mass is equal to $87 \mathrm{~kg} / \mathrm{mol}$. 


\subsection{Blend preparation}

All polymers were vacuum dried, at $50^{\circ} \mathrm{C}$, before melt blending in an internal mixer of the Brabender type. Melt mixing of the various blends was performed at $190^{\circ} \mathrm{C}$ and $50 \mathrm{rpm}$ for 8 min under continuous nitrogen flow to avoid thermal degradation. After processing, the different blends were subjected to a fast quenching in ice water to freeze-in the morphology. Finally, after drying, the blends were annealed for 20 min under a nitrogen blanket at $185^{\circ} \mathrm{C}$. Three ternary blends, with composition 45/10/45 weight \% were prepared (PLA/PCL/PBS, PLA/PBS/PCL, and PCL/PLA/PBS). Moreover, for the sake of comparison, neat polymers were subjected to the same preparation conditions.

\subsection{Blend characterization}

\section{SEM analysis}

A Leica instrument (RM2165) equipped with an LN21 cooling system was used to cryo-microtome blend samples at $-150^{\circ} \mathrm{C}$ and the final morphology was observed at $15 \mathrm{kV}$ using a desktop scanning electron microscope (SEM). The BSE mode (image with backscattered electrons) was used. The 45/10/45 PLA/PBS/PCL blend was stained by 2 wt \% phosphotungstic acid in order to increase phase contrast. Gold coating on the microtomed surface was also employed as needed.

Several micrographs of the most representative inner regions from different blends were acquired. The diameters of the dispersed minor phases were measured via image analysis of at least 100 droplets using a Wacom digitizing table and SigmaScan v.5 software.

\section{Polarized Optical Microscopy}

Polarized Optical Microscopy (PLOM) was used to observe the nucleation step and morphology development of the different components in the various blends. Films with a thickness of about 20-30 $\mu \mathrm{m}$ were obtained by microtoming and by gentle compression on a hot plate between two microscope glass slides. The polarized light optical microscope, Olympus BX51, equipped with an Olympus SC50 digital camera was employed to follow the spherulite development. The applied thermal protocols were controlled by a Linkam TP-91 hot stage. Different components (PLA, PCL, and PBS) were chosen due to their different melting and crystallization ranges, which allows us to study the crystallization of each phase separately. Stepwise crystallization of the different polymer components was performed, according to the thermal protocol shown in Figure S1. The films were firstly held at $200^{\circ} \mathrm{C}$ for 3 minutes to erase the effects of previous thermal history, and then they were quenched to 
the crystallization temperature, where the nucleation and growth of polymer spherulites were monitored. The polymers were crystallized in sequence: PLA $\left(T_{c}\right.$ range $\left.120-130^{\circ} \mathrm{C}\right)$, PBS $\left(105-90^{\circ} \mathrm{C}\right)$, and PCL $\left(42-52^{\circ} \mathrm{C}\right)$. The holding time at each isothermal temperature was always sufficient to complete the primary crystallization of the given component before cooling the sample in the crystallization temperature range of the next lower melting polymer.

\section{Differential scanning calorimetry}

Differential Scanning Calorimetry (DSC) characterization was performed using a Perkin Elmer DSC Pyris 1 calorimeter, equipped with a refrigerated cooling system (Intracooler 2P).

A calibration process was carried out using indium and tin prior to analysis. Samples with a mass of approximately $5 \mathrm{mg}$ were used. During the DSC analysis, a constant nitrogen flow of $20 \mathrm{ml} / \mathrm{min}$ was applied.

Non-isothermal measurements: Samples were melted at $200^{\circ} \mathrm{C}$ for $3 \mathrm{~min}$ to erase the thermal history of all the components and then cooled down to $-20^{\circ} \mathrm{C}$, at cooling rates of $5^{\circ} \mathrm{C} / \mathrm{min}$. Finally, the samples were subsequently heated to $200^{\circ} \mathrm{C}$, at a rate of $5^{\circ} \mathrm{C} / \mathrm{min}$.

Isothermal measurements: different thermal protocols were applied for the analysis of the isothermal crystallization, depending on the overall crystallization kinetics of the considered crystalline component. If the kinetics is directly measurable at the crystallization temperature, the thermal protocol described by Lorenzo et al. ${ }^{[4]}$ was employed. Thus, samples were firstly heated to $200^{\circ} \mathrm{C}$ and held at $200^{\circ} \mathrm{C}$ for $3 \mathrm{~min}$ to erase the thermal history of different components. Subsequently, the sample is quenched at a cooling rate of $60^{\circ} \mathrm{C} / \mathrm{min}$ to the desired crystallization temperature $\left(T_{c}\right)$. The isothermal scan is then recorded. A prior test was performed to detect the minimum $T_{c}$ achievable without the occurrence of any crystallization during the cooling scan. The different polymers were separately crystallized in successive steps. After completion of the crystallization of the higher $T_{c}$ component, the sample is further cooled to the $T_{c}$ of the second (and eventually third) polymer.

The isothermal crystallization behavior of the component with the lower content, i.e., the polymer present at $10 \mathrm{wt} \%$ concentration, was probed by an isothermal step crystallization protocol. After erasing the crystalline history at $200^{\circ} \mathrm{C}$ for $3 \mathrm{~min}$ and quenching the sample at $60^{\circ} \mathrm{C} / \mathrm{min}$ to the chosen $T_{c}$, the sample was held there for a given time, $t_{c}$, and subsequently melted at a rate of $10^{\circ} \mathrm{C} / \mathrm{min}$. The melting enthalpy recorded corresponds to the crystallization enthalpy of the crystals formed during the holding time at $T_{C}$. The procedure 
was repeated for increasingly longer times, until an asymptotic value of the melting enthalpy, for a given crystallization temperature, was reached ${ }^{[45]}$.

\section{Results and Discussion}

\section{Morphological characterization with Scanning Electron Microscopy}

Figure 1 presents SEM micrographs of cryogenically microtomed surfaces of the various ternary blends (PLA/PCL/PBS, PCL/PLA/PBS, and PLA/PBS/PCL with the composition of 45/10/45) after annealing. The prepared ternary blends exhibited a partialwetting morphology in which droplets of the minor component self-assemble at the interface of the other two major components (between the co-continuous structure formed by components with the composition of $45 \mathrm{wt} \%)$. The segregation of the various phases and the obtained morphology confirm the immiscibility of the different components.
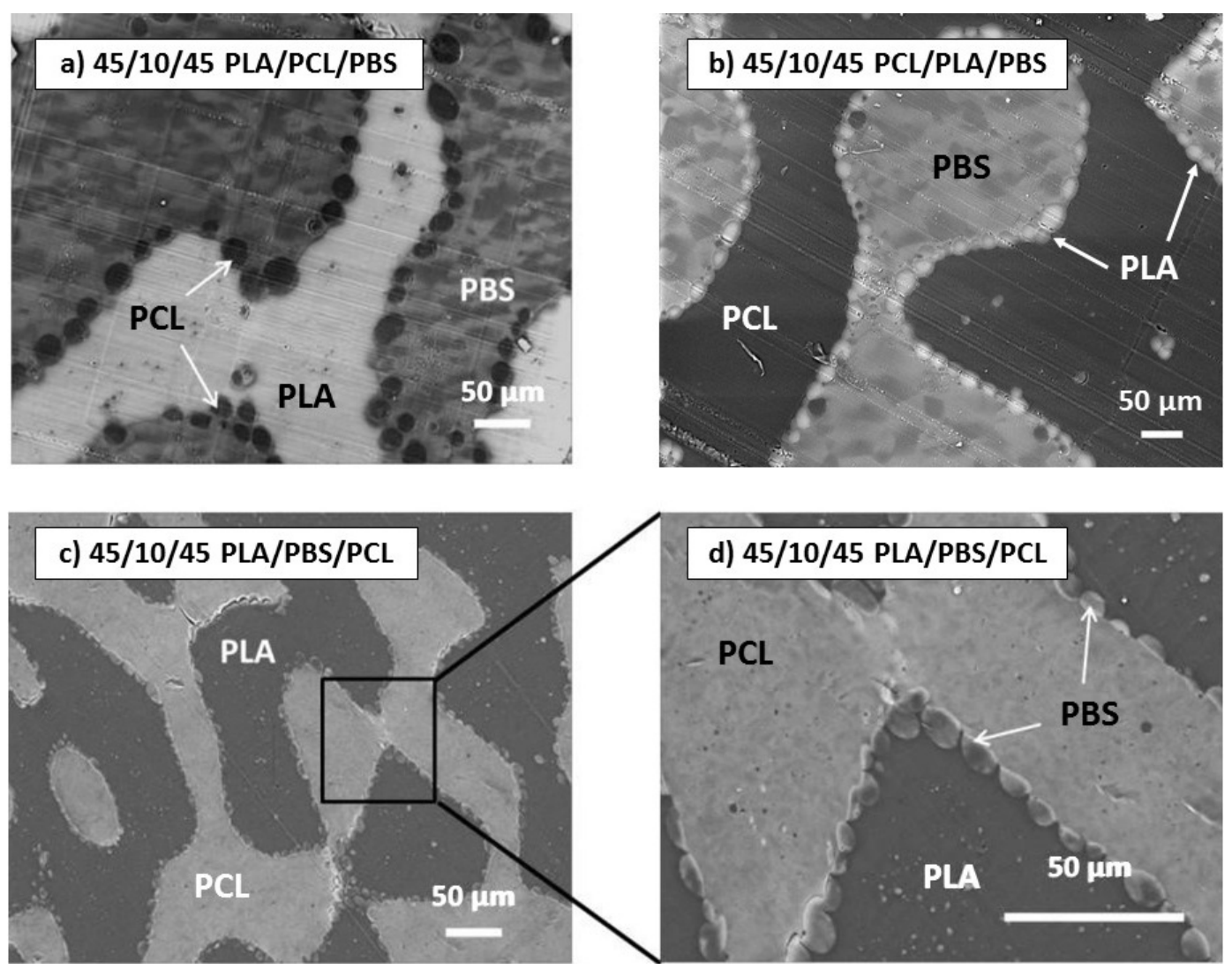

Figure 1. SEM micrographs of PLA/PCL/PBS, PCL/PLA/PBS and PLA/PBS/PCL ternary blends with weight composition of $45 / 10 / 45$ after annealing for $20 \mathrm{~min}$ at $185^{\circ} \mathrm{C}$. a) and b) were directly imaged after cryo-microtoming; c) and d) were stained by tungstic acid followed by gold coating ( $\sim 1 \mathrm{~nm}$ thickness) before SEM analysis. 
The obtained morphology results are in line with those published by Ravati et al. ${ }^{\text {[46] }}$ and Fenni et al. ${ }^{[43]}$ The exact shape of the self-assembled droplets is mainly controlled by the spreading coefficient and the differences in the interfacial tension between different polymer pairs as described by the Neumann Triangle ${ }^{[43,47-48]}$. The self-assembled droplets exhibit asymmetric shapes due the differences in the polymer-polymer interfacial tensions. The different phases in Figure 1 could be identified as follows. By comparing the minor phases at the interface from Figures $1 \mathrm{a}$ ) and b), we can identify that the dark phase is PCL and the bright phase is PLA. The PBS phase shows some textured features. For Figure 1c), it was difficult to clearly define the interface of PCL and PBS, so a tungstic acid solution was used to stain the phases (this completely changed the phase contrast). Since the interfacial tension between PLA and PBS is much lower than that of PBS/PCL, the minor PBS phase tends to be oriented towards the PLA phase. With this morphological configuration, the PLA and PCL phases in Figure 1c can be then identified.

Table 1 reports the percentage of the minor phase located at the interface and the average droplet size in the various blends. The percentage of minor phase at the interface was estimated by using image analysis on the SEM images, i.e., the area occupied by the minor phase at the interface divided by the total area occupied by that phase in the whole image (both at the interface and in the bulk). The diameters of the droplets are crucial and important for the crystallization of the minor component. In our case, the droplets are rather large in all blends (average diameters are about $10 \mu \mathrm{m}$ or higher) which will result in a bulk-like crystallization behavior (it will be unlikely to observe fractionated crystallization phenomena).

Table 1 Composition $(\mathrm{wt} \%)$, domain size (volume average $\left(D_{\mathrm{v}}\right)$ and number average $\left(D_{\mathrm{n}}\right)$ diameters) of the minor phase and its percentage at the interface in the ternary blends.

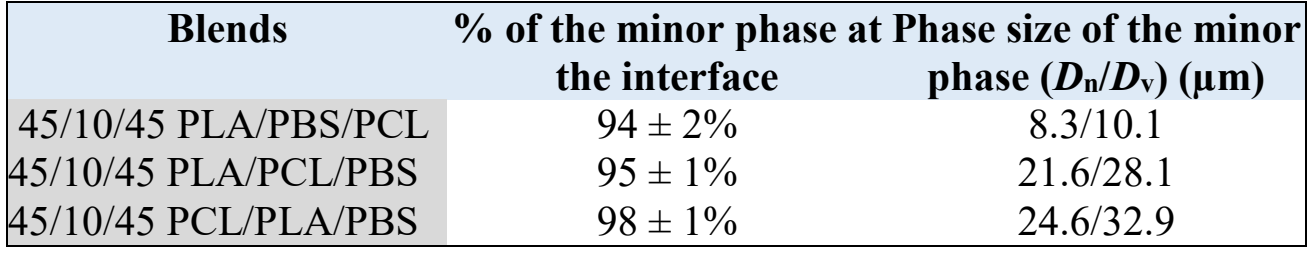

\section{Differential scanning calorimetry}


The DSC cooling and heating scans of the different blends at a rate of $5^{\circ} \mathrm{C} / \mathrm{min}$ are shown in Figure 2, while the characteristic temperatures and enthalpies of the different phases are reported in Tables S1 and S2 of the Supporting Information.
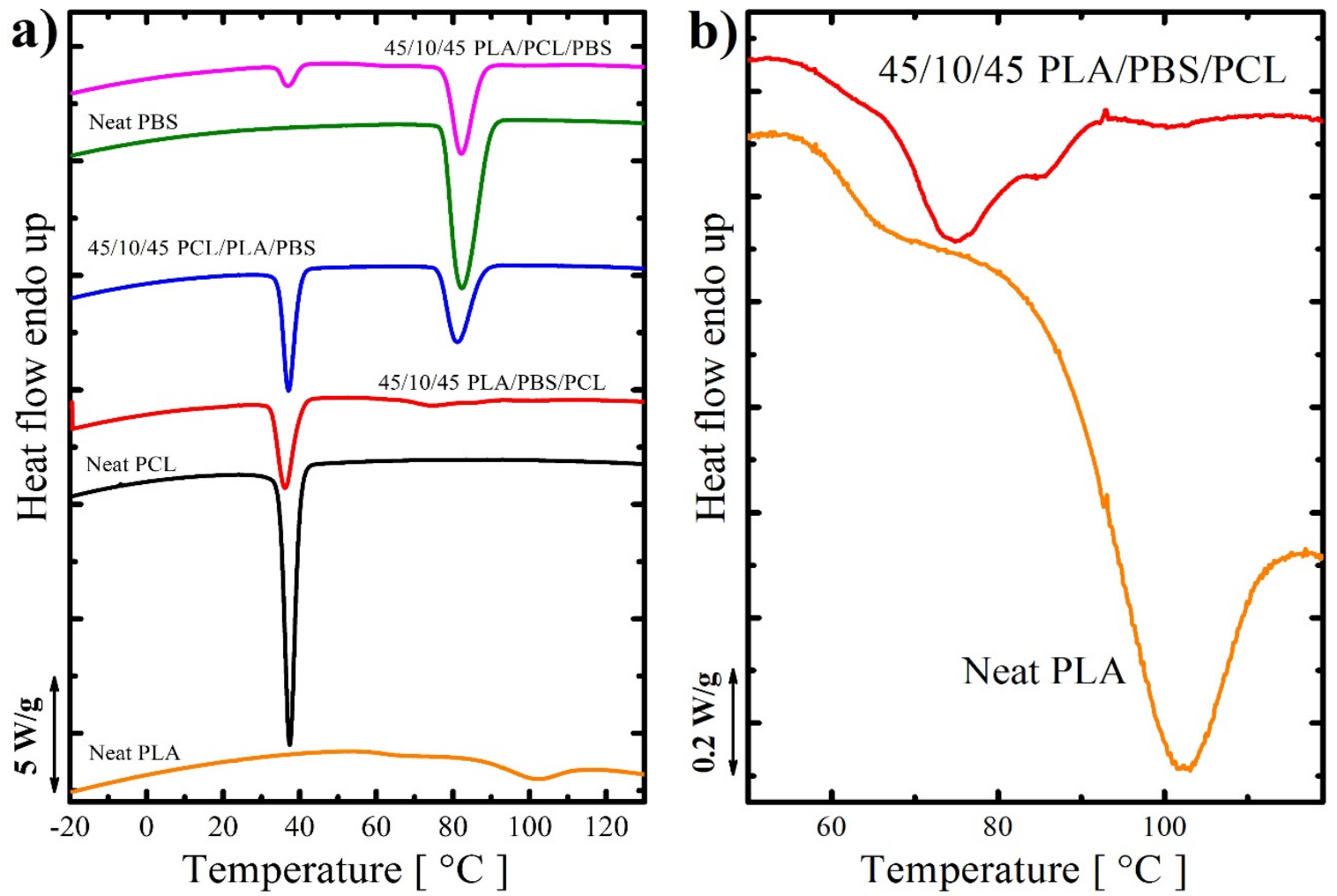


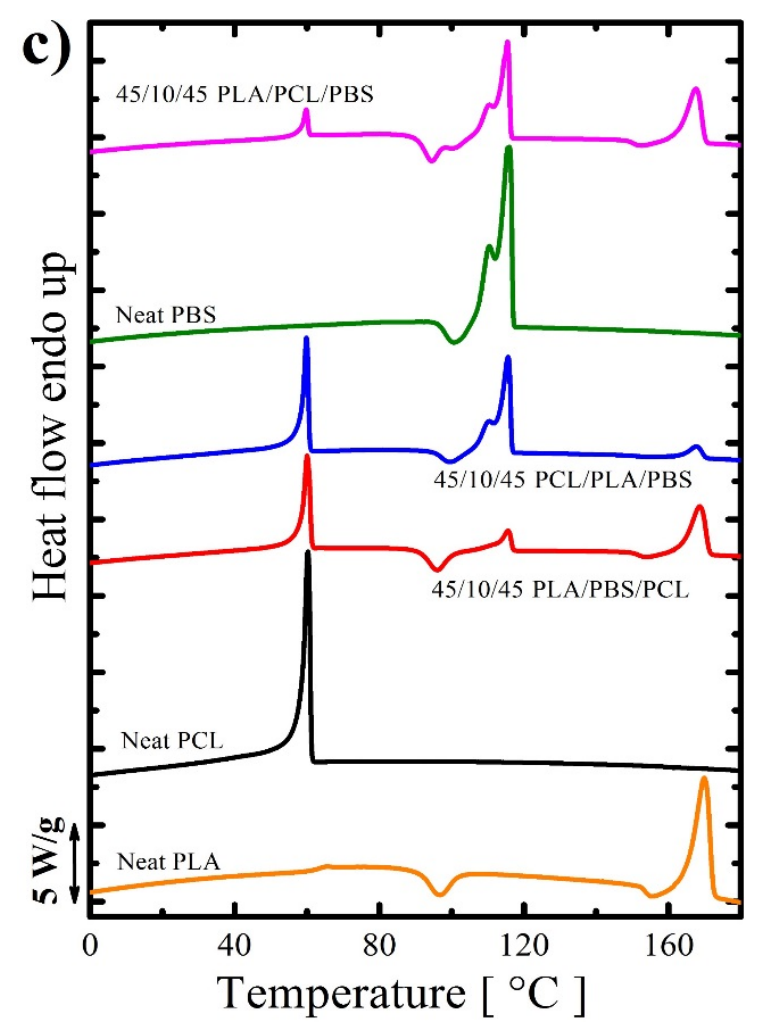

Figure 2. (a) DSC cooling scans, (b) close-up of the crystallization curves of neat PLA and $45 / 10 / 45 \mathrm{PLA} / \mathrm{PBS} / \mathrm{PCL}$ in the range $120-50^{\circ} \mathrm{C}$, and (c) the subsequent heating scans for the neat polymers and the different ternary blends at a scan rate of $5^{\circ} \mathrm{C} / \mathrm{min}$.

Neat PLA crystallizes partially during cooling and exhibits a broad crystallization exotherm which peaks at $101{ }^{\circ} \mathrm{C}$ (Figure 2a). Its crystallization is completed during the heating scan via two cold crystallization steps; the first located at around $97^{\circ} \mathrm{C}$ and a second one that occurs just before melting. This second exothermic event is tentatively related to the recrystallization of PLA disordered $\alpha^{\prime}$-phase into the more stable $\alpha$-crystals ${ }^{[49]}$. Finally, PLA melts at about $170^{\circ} \mathrm{C}$ (Figure 2c). PCL exhibits a single crystallization peak at around $37^{\circ} \mathrm{C}$ and shows a sharp melting endotherm which peaks at around $60^{\circ} \mathrm{C}$. Neat PBS shows a crystallization exotherm located at $83^{\circ} \mathrm{C}$, while on heating it presents a small exotherm just before $116^{\circ} \mathrm{C}$. This polymer is characterized by a bimodal melting peak which could be the result of a reorganization process of the lamellar crystals ${ }^{[50]}$.

The DSC cooling and heating scans of the different polymers in the ternary blends are not dramatically different from those of the neat polymers once the relative content of each phase is taken into account. In particular, it seems that the melt mixing process does not significantly influence the crystallization behavior of both PCL and PBS, in all studied ternary blends. In fact, the crystallization temperature is practically identical to the one of the neat components. Similar considerations apply to the melting process of these two phases. On 
the other hand, the PLA phase within 45/10/45 PCL/PLA/PBS and 45/10/45 PLA/PCL/PBS ternary blends does not show any evident crystallization exotherm during the cooling scans. It must be deduced that nucleation is delayed in these blends, possibly due to transfer of the most efficient nucleating heterogeneities from PLA to the other phases, during mixing at high temperatures ${ }^{[19,48]}$. On the other hand, in 45/10/45 PLA/PBS/PCL, a small crystallization exotherm can be observed (see Figure $2 b$ ), although at lower temperatures with respect to that of neat PLA.

DSC heating scans suggest that the cold crystallization behavior of the PLA phase within 45/10/45 PLA/PCL/PBS and 45/10/45 PLA/PBS/PCL is slightly shifted toward lower temperatures with respect to the one of the neat polymer, suggesting a mild nucleating effect of PCL and PBS. A similar enhancement of PLA cold-crystallization by the presence of these two immiscible polymers has been previously reported in the literature ${ }^{[12,48,50-52]}$. However, we note that due to the overlap with the PBS re-organization and melting region, the cold crystallization enthalpy of PLA in the ternary blends, is not accurately measurable.

The crystallization kinetics of the blended samples, was further investigated under isothermal conditions, either by directly measuring the crystallization exotherm as a function of time at $T_{c}$, or with stepwise-crystallization experiments, when the considered phase was at low concentration ${ }^{[44-45]}$. The obtained isothermal crystallization data from DSC was analyzed by the Avrami equation, with the data fitting performed by using the Origin ${ }^{\circledR}$ plug-in developed by Lorenzo et al. ${ }^{[44]}$ An example of the fit obtained for the different neat polymers and their blends, at selected crystallization temperatures, is shown in Figure S2 of the Supporting Information.

The inverse of half-crystallization time $\left(1 / \tau_{50 \%}\right)$, which is a measure of the overall crystallization rate (comprising contributions from both nucleation and growth) for the PLA, PCL, and PBS phases in the different systems, is reported as a function of crystallization temperature $\left(T_{c}\right)$ in Figures $3 \mathrm{a}, 3 \mathrm{~b}$ and $3 \mathrm{c}$, respectively. The Avrami exponents $(n)$, containing information on the growth dimensionality and nucleation modality, obtained for the different crystallizing phases and conditions, is instead shown in Figures S3a through S3c of the Supporting Information.

Figure 3a shows the overall crystallization rate of neat PLA and of the PLA phase in the different ternary blends, where PLA is either one of the major phases or the minor component. The crystallization rate of PLA increases with decreasing $T_{c}$, both for neat PLA and for all the ternary blends as expected in this low supercooling range. 
With respect to neat PLA, a lower crystallization rate of that same polymer in the blends can be observed in the whole crystallization temperature region. This effect is consistent with the previously discussed non-isothermal data. It should be noted however that the difference in crystallization rate with respect to the unmixed polymer is only minor, at most a factor two. Again, since the polymers are immiscible, the crystal growth rate is expected to be unaffected by the blending process ${ }^{[51-52]}$, and hence the observed decrease is attributed to the transfer of nucleating impurities from the PLA to the other polymers during mixing, which brings about the decrease in the concentration of active heterogeneous nuclei.
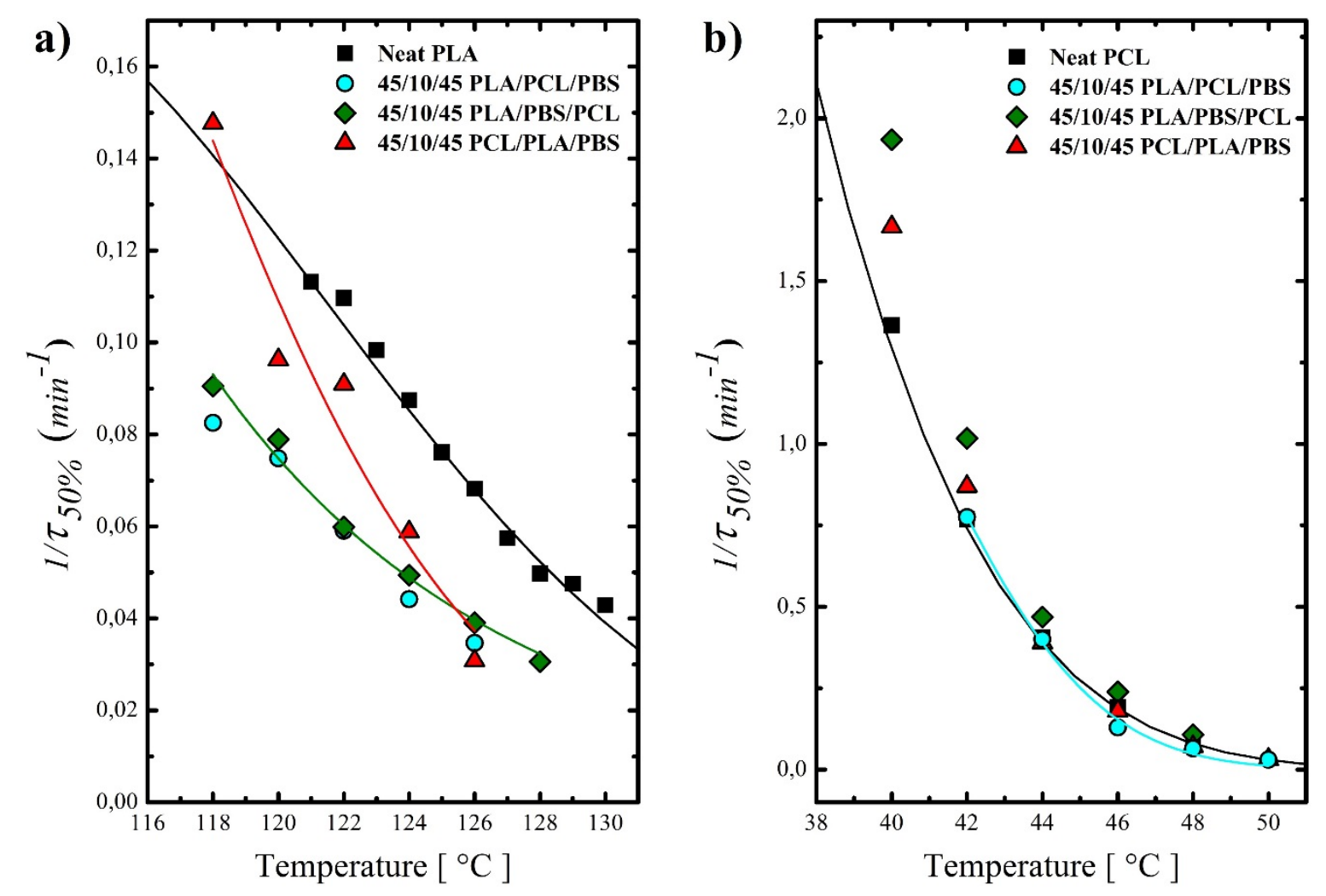


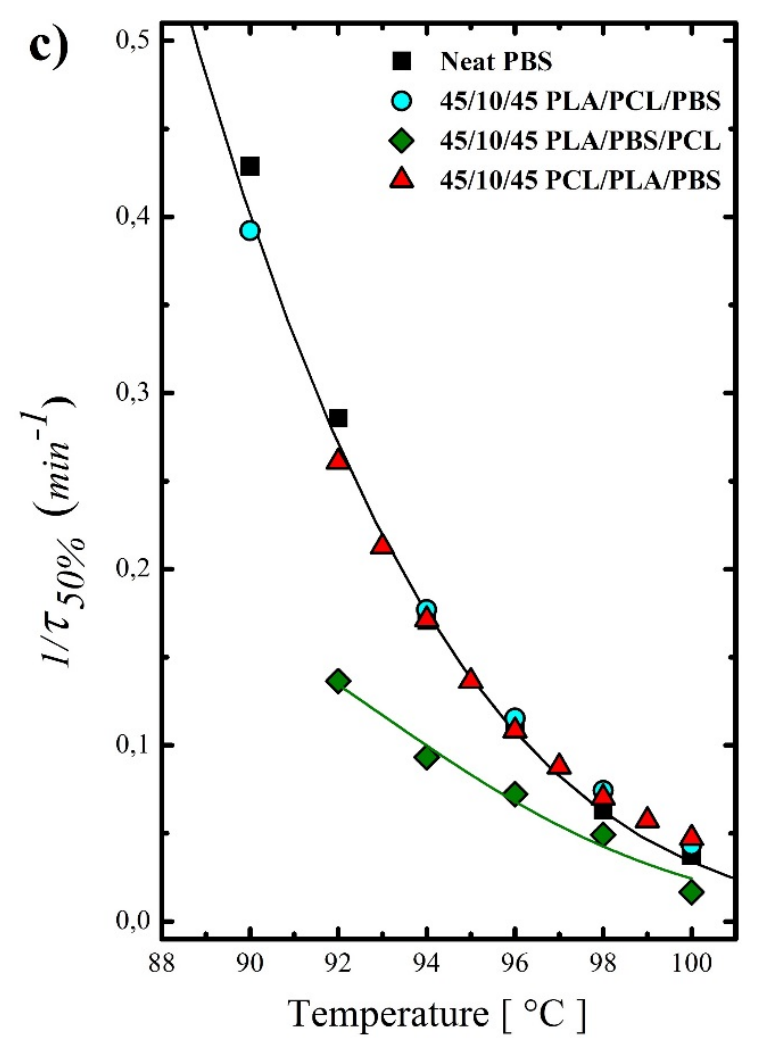

Figure 3. Reciprocal of the half-crystallization time $\left(1 / \tau_{50 \%}\right)$ for a) PLA, b) PCL, and c) PBS in the different blends, as a function of crystallization temperature. Black continuous lines represent fits of the overall crystallization rate data of the neat polymers according to the Lauritzen-Hoffmann model. Colored lines instead are obtained by fitting the data point with an exponential law, and are added to guide the eyes.

The Avrami exponent of the neat PLA and the PLA phase within different blends is always found to be between 2 and 3, regardless of the specific sample or crystallization temperature (Figure S3a). The obtained results suggest that the nucleation mechanism of PLA in all the studied blends can be well represented by the instantaneous nucleation of 3-D spherulites ${ }^{[20-21,52]}$. The analysis of the crystallization enthalpy evolution (Figure S2a) shows a typical sigmoidal trend as well, revealing that the droplets are large enough to allow bulklike crystallization of the PLA phase, contrary to what is observed in systems with crystallizing isolated sub-micron size droplets, which display a first-order crystallization kinetics ${ }^{[7]}$. The observed exponent is also compatible with the existence of a possible interconnection between the PLA domains, which would allow for crystallization proceeding from one droplet to the others (see the Discussion part).

The overall isothermal crystallization kinetics of neat PCL and PCL in ternary blends at the different crystallization temperatures are shown in Figure 3b. All samples exhibit very similar crystallization kinetics in the explored temperature range. A slight increase in the 
overall crystallization rate of PCL can be observed for the ternary blends containing $45 \mathrm{wt} \%$ of PCL. This small enhancement could be related to a mild nucleating effect of the previously crystallized PBS or PLA droplets on the undercooled PCL phase. PCL droplets within 45/10/45 PLA/PCL/PBS ternary blend exhibit overall crystallization rate analogous to that of neat PCL, given the relatively large average size of PCL domains, which leads to a high fraction of droplets containing the same nucleating heterogeneities responsible for the nucleation of bulk PCL. Also for PCL, the values of the Avrami index (n) (Figure S3b) are close to 3 and practically independent of the crystallization temperature, suggesting a crystallization mechanism based on the instantaneous growth of 3-D spherulites. For the ternary blend where PCL is the minor component, the collected experimental data were limited in certain conditions and did not allow for an accurate determination of the Avrami exponent (see the crystallinity evolution of this system in Figure S2b). As such, the Avrami exponents are not reported, although values around 2 are obtained when sufficient experimental data at low crystallization time are available.

The results related to the crystallization of the PBS phase are similar to those obtained for PLA and PCL in the different blends (Figure 3c). In particular, the crystallization kinetics of PBS major phases, in the 45/10/45 PLA/PCL/PBS and 45/10/45 PCL/PLA/PBS blends, is practically unchanged with respect to that of neat PBS. While a small decrease in the overall crystallization rate is observed for the PBS droplets in the 45/10/45 PLA/PBS/PCL ternary system. The observed decrease could be related to the lack of active heterogeneities responsible inside each single PBS droplet. On the other hand, the Avrami exponent was found to be relatively unchanged by the blending process and is close to 3 for all the crystallization conditions (Figure S3c), when PBS is one of the major phases, as expected for the heterogeneous nucleation and growth of 3D spherulitic morphologies. When PBS is present in the form of droplets in ternary blends, the same considerations on Avrami fitting put forward for the case of the PCL system apply.

The detailed DSC analysis has revealed only minor differences in the overall crystallization kinetics of the different polymers in ternary blends compared to neat materials, the crystallization process is further explored by means of polarized optical microscopy, which enables the direct visualization of the nucleation modalities in the different systems. 


\section{PLOM study of isothermal crystallization}

The development of crystalline morphologies in the tri-crystalline ternary blends was studied by means of PLOM, by examining the sequential crystallization of each of the polymer phases in a different temperature range, according to the thermal protocol described in Figure S1.

\section{Crystallization of the major phases in immiscible ternary blends with partial-wetting morphology}

The possible effect of the presence of the partially-wet droplets on the crystallization of the major co-continuous phases will be investigated in this part. Due to the exceedingly high nucleation density of the PCL phase, attention will be focused on the crystallization of the PLA and PBS phases only whose morphology is particularly suitable for PLOM investigation.

Figure 4 shows PLOM micrographs taken at different times during the isothermal crystallization of PLA at $120^{\circ} \mathrm{C}$ in $45 / 10 / 45$ PLA/PBS/PCL (a-c) and 45/10/45 PLA/PCL/PBS (d-f). Experiments referring to the different systems are arranged in two columns: on the left the ternary blend with PBS droplets at the interface between PLA and PCL is presented, while on the right PCL minor phase located between PLA and PBS phases is shown. In each row, the same crystallization time for the two different samples is displayed.

45/10/45 PLA/PBS/PCL

45/10/45 PLA/PCL/PBS

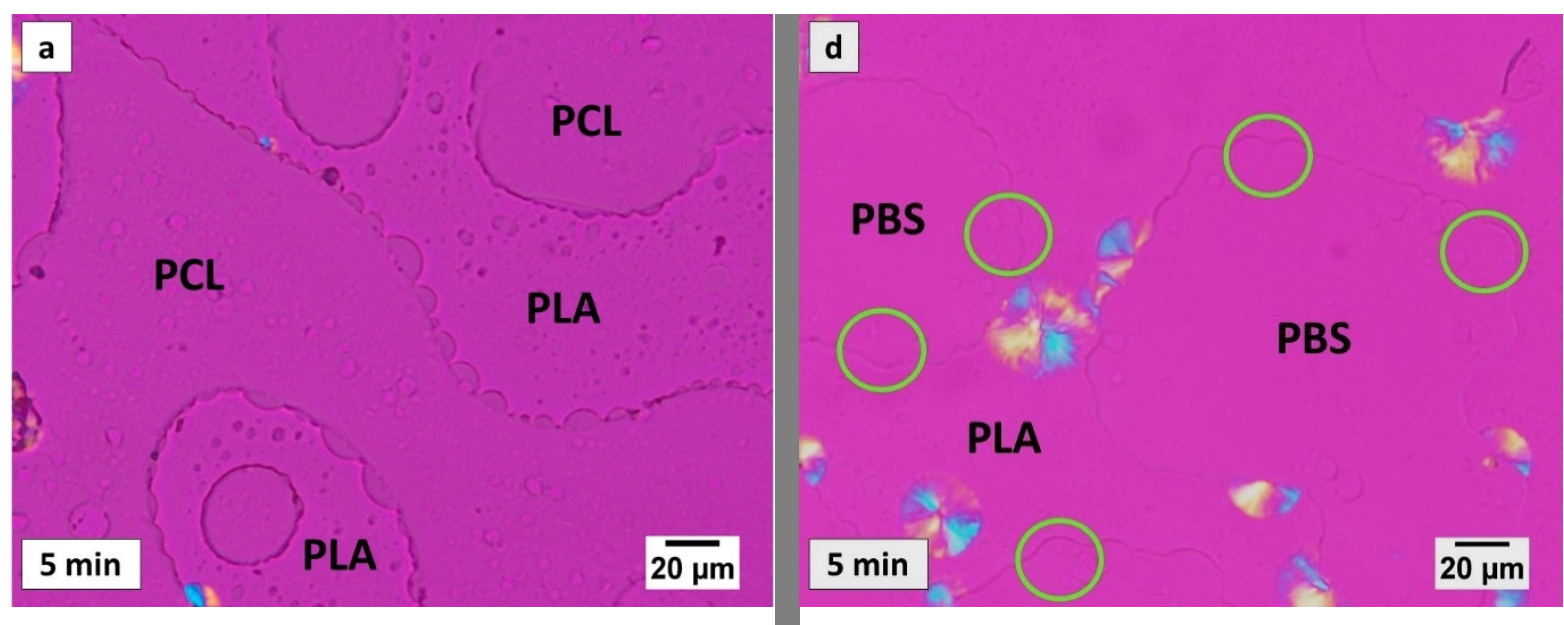




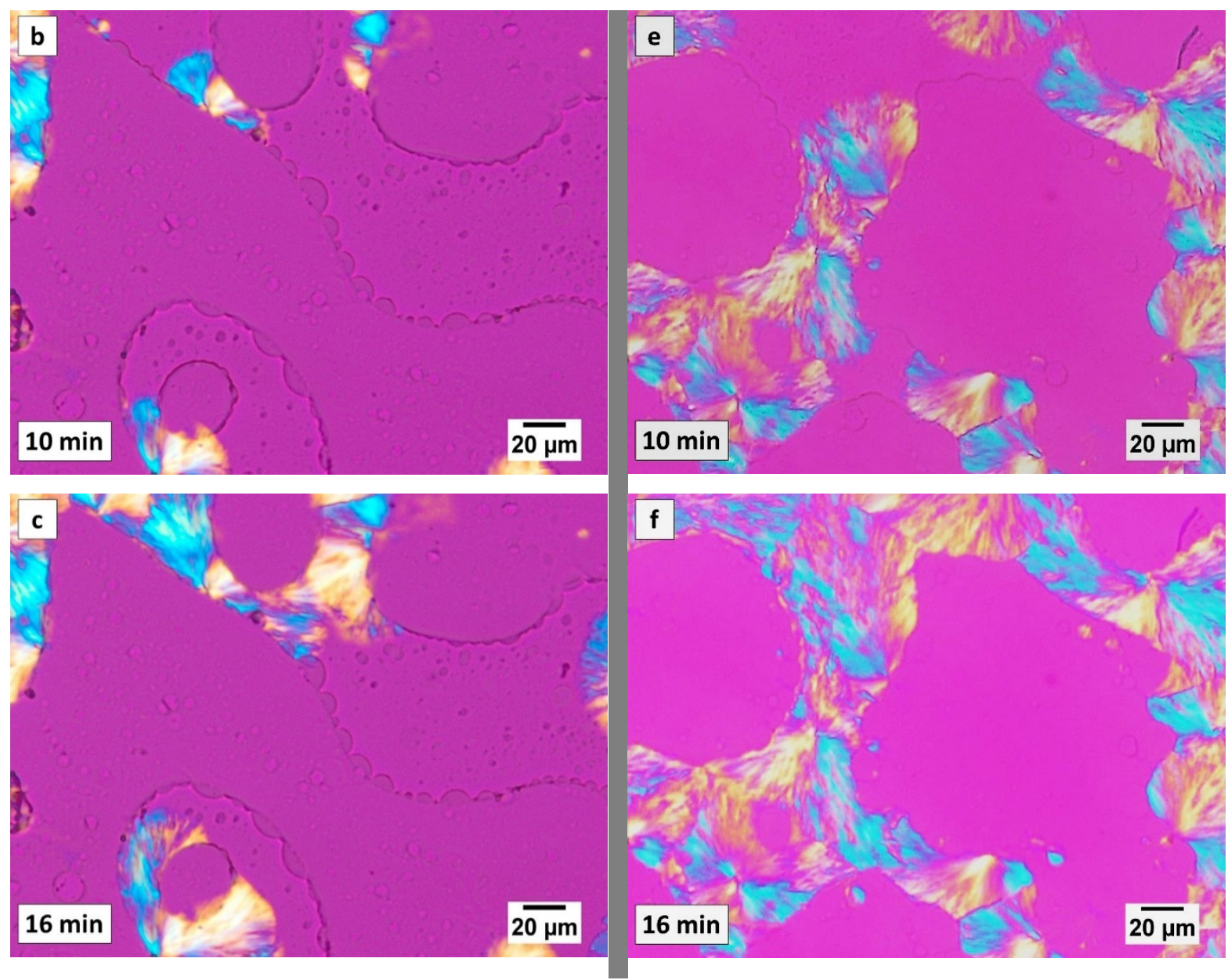

Figure 4. PLOM micrographs taken during the isothermal crystallization of PLA at $120^{\circ} \mathrm{C}$ at the indicated times for two different ternary blends: a-c) 45/10/45 PLA/PBS/PCL, d-f) 45/10/45 PLA/PCL/PBS. The green circles highlight some locations where molten PCL droplets at the interface between PLA and molten PBS are present.

PLOM micrographs reveal that the PLA major phase, in both ternary blends, preferentially nucleates at the interface with the other molten components. Given the high coverage of the close-packed droplet array in these partial wetting morphologies (as derived by SEM morphological analysis), one can reasonably assume that nucleation occurs at the interface with the molten droplet minor phase, i.e., on PBS or PCL in PLA/PBS/PCL and 45/10/45 PLA/PCL/PBS blends, respectively. This interfacial nucleation modality dominates the obtained morphology since only a limited number of nuclei are formed in the bulk of the PLA phase.

By comparing the different ternary blends, i.e., Figure 4a-c and d-f, a slight preference of PLA for the nucleation on molten PCL droplets can be deduced. In fact, after equivalent crystallization time the number of surface-nucleated PLA spherulites in the ternary 45/10/45 PLA/PCL/PBS is higher than that in the 45/10/45 PLA/PBS/PCL blend. Moreover, the size 
of PLA spherulites at a given time is larger for the second sample (Figure 4d-f), which means nucleation occurs at earlier times in the 45/10/45 PLA/PCL/PBS blend. Therefore, under the assumption that the surface-nucleation events highlighted in Figure 4 are actually occurring at the interface with partially-wet droplets, and considering the interfacial areas with PLA are similar in the two blends (or higher for the 45/10/45 PLA/PBS/PCL, as expected on the basis of the interfacial tensions for PBS/PLA and PCL/PLA pairs ${ }^{[53-54]}$ ), these results on the nucleation of the PLA major phase confirm the higher apparent nucleation efficiency of molten PCL surfaces with respect to the PBS melt. Similar results have been previously reported by us for PLA partially-wet droplets in the 45/10/45 PBS/PLA/PCL blend ${ }^{[43]}$.

A further demonstration of the occurrence of PLA nucleation on the contact surface with molten PBS or PCL can also be found by analyzing the crystallization of PLA droplets in binary blends with the two polymers in-situ. PBS/PLA and PCL/PLA blends with 90/10 compositions have been prepared by batch melt-mixing under the same conditions described for the ternary blends (Material and Methods section). Figure S4 in the Supplementary Information shows PLOM micrographs taken during the crystallization of PLA droplets within 90/10 PBS/PLA blends at $120^{\circ} \mathrm{C}$. The droplets have undergone a substantial coarsening during the melting process, reaching an average diameter larger than 10 micrometers, suitable for the optical microscopy observation. PLA spherulites are clearly seen to nucleate and grow in the separate PLA domains until each droplet is completely crystallized. The point at which the nucleation occurs can be distinguished in most of the domains. Although a certain fraction of the PLA spherulites apparently originate from the bulk of the droplet, nucleation at the interface between molten PLA and PBS can be seen in several cases, as highlighted by the arrows. Unfortunately, a similar direct morphological observation cannot be provided for the 90/10 PCL/PLA blend, since the PLA droplet size is too small to be discernible, and an appropriate coarsening of the morphology with mild meltannealing treatments could not easily be obtained.

The observed PLA interfacial-induced nucleation at the interface with molten PCL and PBS interfaces in the 45/10/45 PLA/PCL/PBS and 45/10/45 PLA/PBS/PCL blends can be explained through several theories, the most plausible one is the effect of impurities migrating to the interface between components during the melt mixing step. Other alternative interpretations are considered below.

Previous studies revealed the enhanced nucleation of polyolefins in their amorphous/crystalline blends upon fluctuation-assisted nucleation near the interface ${ }^{[55-56]}$ and 
the obtained results could be interpreted using the following two theoretical frameworks [57$58]$.

$\mathrm{Hu}$ et al. demonstrated, using dynamic Monte Carlo simulations, that the crystal nucleation near the interface is slightly enhanced in immiscible binary blends. The authors attributed the observed enhancement to the increase of the equilibrium melting point of the crystals upon dilution therefore leads to a local increase of the supercooling, at a given $T_{c}$, which faster and favor the nucleation at the interface. For a more detailed explanation please refer to $\mathrm{Hu}$ et al. ${ }^{[57]}$ and Fenni et al. ${ }^{[43]}$

An alternative explanation was proposed by Muthukumar et al. [58] The authors describe the effect of the spinodal decomposition in forming spontaneously interface and domains which can play the role of heterogeneous nucleation surface for the crystalline component.

The observed preferential nucleation on PCL surfaces can be explained according to our previous results, in which we interpret it in terms of interfacial free energy difference which has been found to be slightly lower for PLA/PCL $(19.2 \mathrm{Nm} / \mathrm{m})$ than for PLA/PBS $(19.9 \mathrm{Nm} / \mathrm{m})$. More details on the calculation of the interfacial free energy difference can be found in our previous work ${ }^{[43]}$

Next, the crystallization of the PBS major phase in the ternary blend is considered. The first ternary blend studied is 45/10/45 PLA/PCL/PBS, in which the PLA phase is crystallized first at $120^{\circ} \mathrm{C}$ for $20 \mathrm{~min}$, and the isothermal crystallization of PBS at $100^{\circ} \mathrm{C}$ then follows. Figure 5 shows selected PLOM micrographs captured during PBS crystallization in the presence of solidified PLA. The crystallization of the PBS domains clearly starts from its edges, and the crystals grow towards the inner part of this major phase. A strong nucleation effect of the interface is thus apparent from the development of the PBS morphology. In fact, because of the high "linear nucleation density" at the interface, the growth of PBS spherulites can only occur perpendicular to the PLA/PBS interface with limited lateral splaying, and a kind of "transcrystalline layer" is formed (Figure $5 \mathrm{~b}$ and c). 

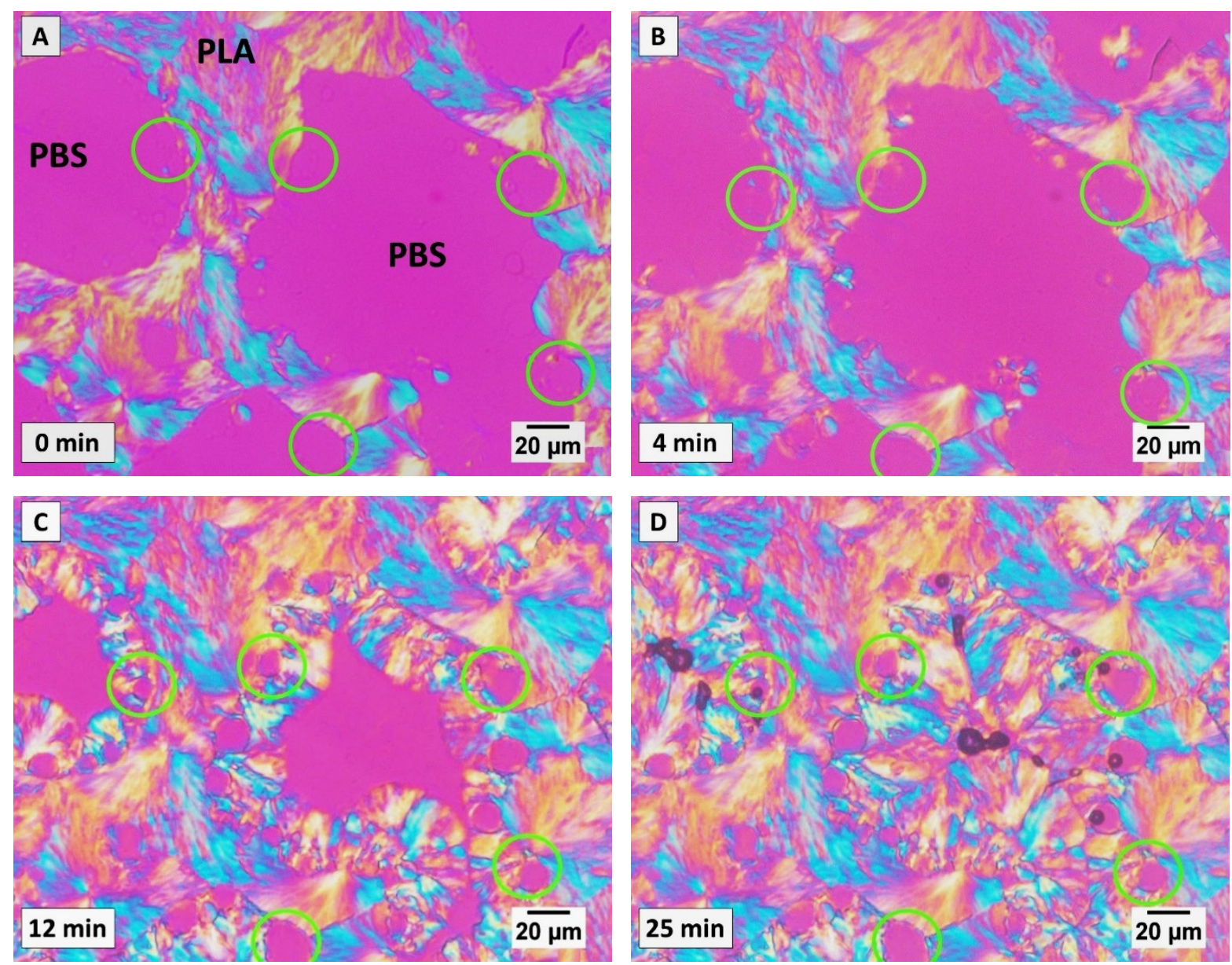

Figure 5. Examples of PLOM micrographs at selected times during the isothermal crystallization of PBS at $T_{c}=100^{\circ} \mathrm{C}$ in the $45 / 10 / 45 \mathrm{PLA} / \mathrm{PCL} / \mathrm{PBS}$ ternary blend. The PLA major phase has been previously crystallized at $120^{\circ} \mathrm{C}$. The green circles highlight some areas when molten PCL droplets at the interface between PLA and PBS can be observed.

Contrary to the previous cases of PLA major phase nucleation at the interface with molten droplets of PCL or PBS in ternary blends (Figure 4), here it seems more plausible that PBS nucleation does not occur at the interface with molten PCL. Rather, it occurs on the semicrystalline PLA surfaces which are exposed to the undercooled PBS melt in the interstitial spaces between PCL droplets at the interface. This can be deduced by observing that the molten PCL domains are mainly engulfed by the PBS transcrystalline layer growth front (Figure $5 \mathrm{c}, \mathrm{d}$ ). In order to support the claim of PBS nucleation on semicrystalline PLA surfaces, the 90/10 PBS/PLA binary blend, can again be considered. Figure S5a presents a micrograph taken during the isothermal crystallization of the PBS matrix, in the presence of previously crystallized PLA droplets. Some of the PBS crystallization is found to start at the interface with semicrystalline PLA (see the black arrow in Figure S5a). Given the fast PBS 
crystallization and relatively high bulk nucleation density of this polymer, it is not easy to distinguish other nucleation events at the polymer/polymer interface.

The fact that the nucleation of PBS on molten PCL is not particularly favored can also be evidenced when considering PBS crystallization in the 45/10/45 PCL/PLA/PBS ternary blend. Examples of micrographs taken at the initial stages of PBS isothermal crystallization, after having completely crystallized the PLA partially wetted droplet, are shown in Figure S5b-d of the Supporting Information. It can be seen that the majority of PBS spherulites originate from the bulk of the PBS phase (highlighted with green circles in the Figure S5b), while only a few of them nucleate in contact with solidified PLA droplets (Figure S5c-d). Nucleation in between PLA domains, i.e., at the molten PCL surface, although admittedly difficult to identify, does not seem to be relevant.

It is worth noting that heterogeneous nucleation in immiscible blends can occur both at molten surfaces (when the adjacent phase is still in the melt state) ${ }^{[14-15,43]}$ and solid surfaces (when the crystallizing polymer is in contact with a previously crystallized component) ${ }^{[16,18,59]}$.

\section{Crystallization of the minor phase in immiscible ternary blends with partial-wetting morphology}

After having presented the results of the crystallization of the major phases in immiscible ternary blends with the partial-wetting morphology, the effect of such major phases on nucleation and crystallization of the partially wet droplets will be discussed.

The results of the isothermal crystallization of PBS droplets in the 45/10/45 PLA/PBS/PCL blend are shown in Figure 6. According to the stepwise crystallization protocol, PLA was firstly crystallized at $125^{\circ} \mathrm{C}$ for 45 min then the sample was quenched to the crystallization temperature of $\mathrm{PBS}\left(100^{\circ} \mathrm{C}\right)$. 

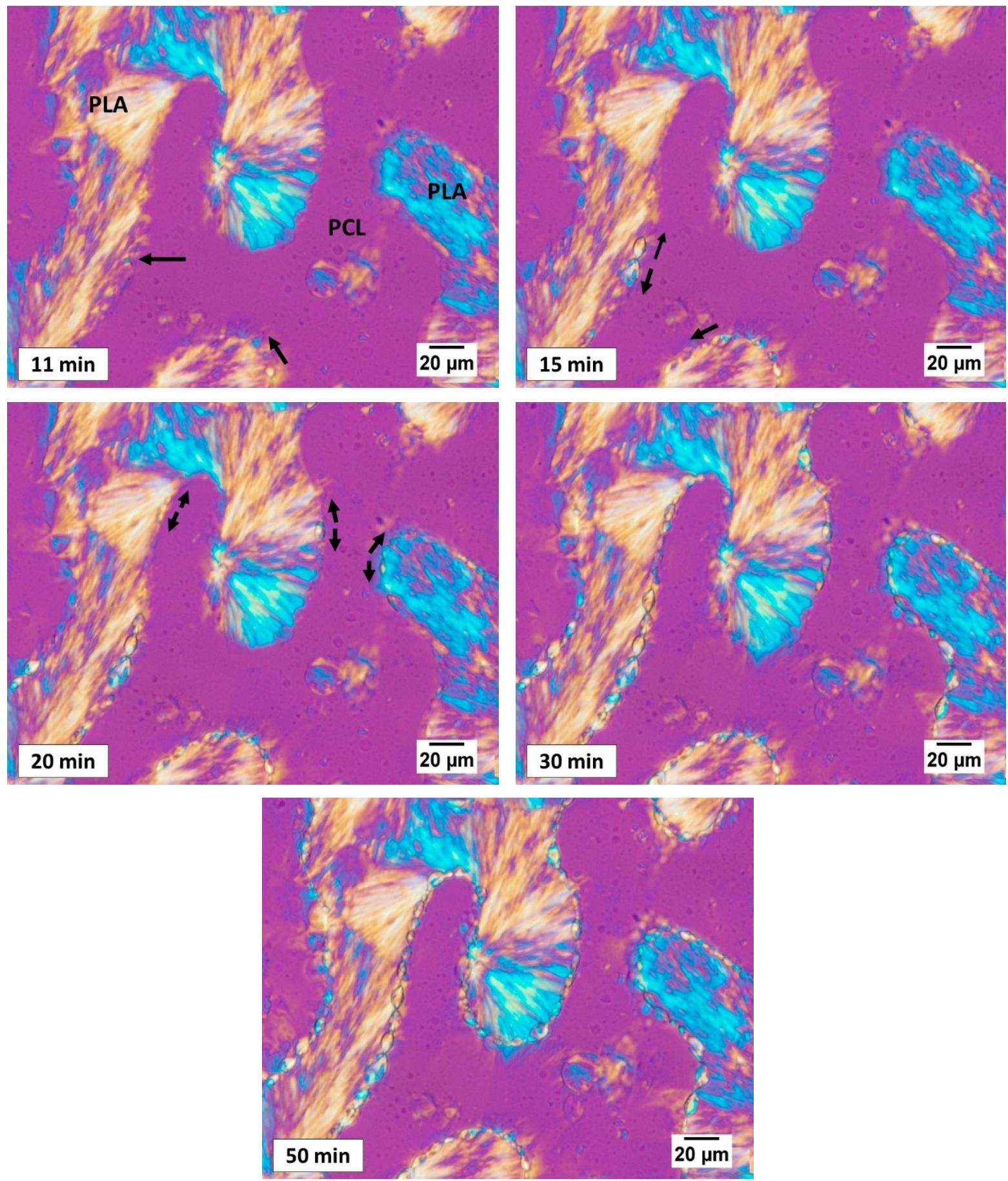

Figure 6. PLOM micrographs during crystallization of $\mathrm{PBS}$ droplets at $100^{\circ} \mathrm{C}$, in the 45/10/45 PLA/PBS/PCL blend, after the indicated times. The arrows indicate the direction of nucleation.

Although the particularly small PBS droplets located at the interface between crystallized PLA and molten PCL are not easily distinguished before crystallization, they become clearly visible upon solidification, due to the development of birefringent spherulites. Similarly to what has been reported by Fenni et al. [43] (PLA droplets in the 45/10/45 
PCL/PLA/PBS ternary blend), it is confirmed that the crystallization of a given droplet can affect the nucleation in adjacent molten domains. Crystallization starts from different droplets, located far apart in the sample. Then, nucleation spreads from droplet to droplet, crystallizing small rows of PBS domains in a sequence. Finally, a three-dimensional crystalline array morphology (pearl-necklace crystalline morphologies in our 2D micrographs), constituted of PBS crystalline droplets around solidified PLA and molten PCL domains, is formed at later stages.

Virgilio et al. ${ }^{[60]}$ reported that droplets of the minor components in immiscible ternary blends with partial-wetting morphology form a perfectly segregated close-packed array at the interface of the co-continuous morphology made by the other two major components. On the other hand, the partially wet droplets are found to not be in contact with each other. This latter observation has been demonstrated by several morphological analyses and the distance between droplets was found to be in the order of 50 nanometers ${ }^{[60]}$.

Figures 1, 4(a-c), and 6, showed that PBS droplets are stable at the PLA/PCL interface and the system exhibits a partial wetting morphology. Looking to the experimentally determined values of polymer/polymer interfacial tension and the calculated spreading coefficient value $\left(\lambda_{\mathrm{PLA} / \mathrm{PBS} / \mathrm{PCL}}=-0.58 \mathrm{mN} / \mathrm{m}^{[43]}\right)$, which is negative but close to zero, one can predict a weak partial wetting regime for this system. On the other hand, Wang et al. ${ }^{[33]}$ reported that the annealing of concentrated partially wet droplets can result in a partial to complete wetting transformation by forming thin completely wet layers, which in time tend to de-wet and return to the initial thermodynamically stable partial wetting morphology.

Such a partial-complete-partial transition can occur, exclusively, in the melt state (when all components of ternary blends are molten). The de-wetting rate depends on the value of the spreading coefficient, which is very close to zero in the studied system, thus the de-wetting rate here will be very slow in comparison to the partial to complete transition rate.

The "quasi stable" thin layer that thus forms during melting will connect, to a given extent, the PBS droplets during crystallization. This allows the nucleation to spread in a sequential mode, and thus explains the observed unusual appearance of nuclei. In other terms, the formed thin layers will act as bridges which guide and orient the nucleation and crystallization events.

On the other hand, during crystallization of the PBS partially wet droplets in our 45/10/45 PLA/PBS/PCL system, the PLA major phase is already crystallized, and the PBS 
droplets are thus completely immobilized at the interface. This eliminates the possibility of the spreading of nucleation through droplet motion or contact.

Such a spreading of nucleation phenomenon was previously investigated and explained for the case of PLA droplets in the 45/10/45 PCL/PLA/PBS ternary blend ${ }^{[43]}$.

The spreading of nucleation and crystallization of the PBS partially-wet droplets resembles the percolation of nuclei observed in interconnected morphologies. Similar nucleation phenomena have been observed in various types of morphologies and systems such as in lamellar or cylindrical micro-domains in segregated block-copolymers, in cocontinuous phases, polymers confined in cylindrical nanopores connected by a polymer layer, and in immiscible blends, including in ternary blends ${ }^{[7,43,61]}$. As such, the isothermal crystallization kinetics exhibit a sigmoidal evolution of the crystallinity in isothermal conditions (see Figure S2 of the Supporting Information), instead of the first-order increase which characterizes crystallization occurring in separated domains.

Finally, we report on the isothermal crystallization experiments of PCL droplets at the interface with crystalline PLA and PBS major phases. Some examples of PLOM micrographs during crystallization of the PCL phase are reported in Figure 7. Although about $90 \%$ of the field of view is occupied by crystalline PLA and PBS domains, thus hindering a clear visualization of PCL droplet crystallization, the solidification of a few domains at different times can be observed. In this third case of a ternary blend with partial-wetting morphology, the spreading coefficient value $\left(\lambda_{\mathrm{PBS} / \mathrm{PCL} / \mathrm{PLA}}\right)$ is equal to $-4.18 \mathrm{mN} / \mathrm{m}$ which indicates a strong partial-wetting regime [43]. Hence no evident droplet-to-droplet spread of the nucleation event, or orientation correlation between neighboring spherulites in the PCL droplets, can be seen. The droplet solidifies as isolated microdomains, in a random-fashion.
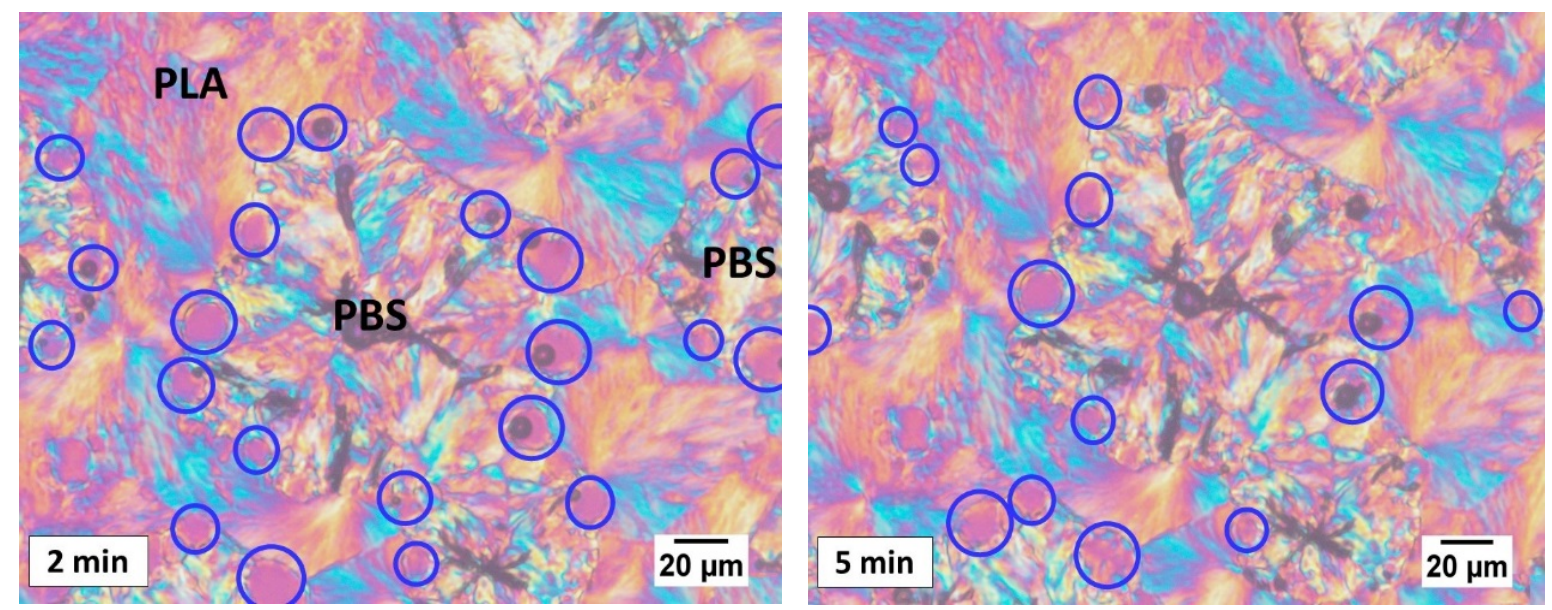

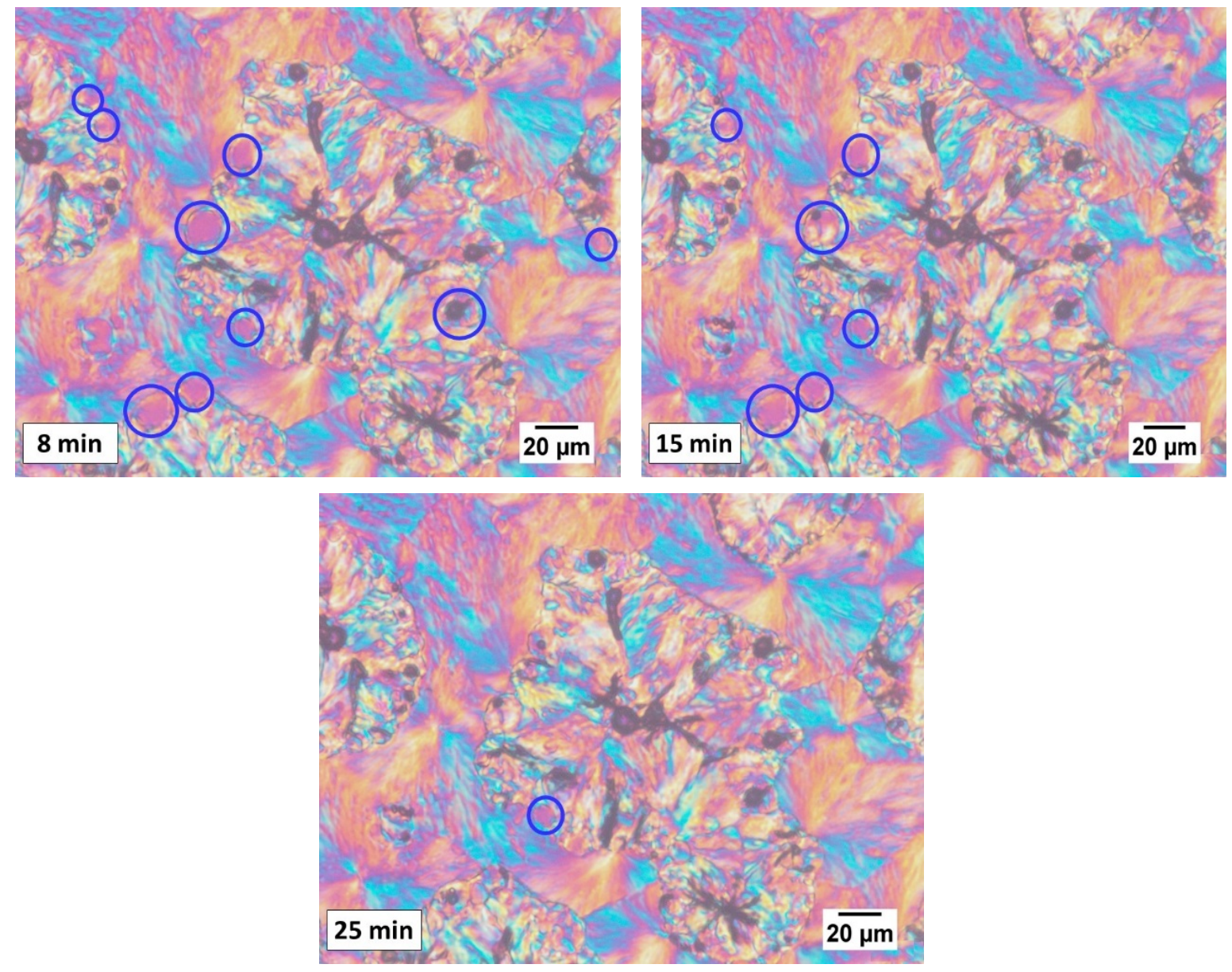

Figure 7. PLOM micrographs taken during the crystallization of PCL droplets at $42^{\circ} \mathrm{C}$, in the $45 / 10 / 45 \mathrm{PLA} / \mathrm{PCL} / \mathrm{PBS}$ blend, after the indicated times. Molten PCL droplets are highlighted with blue circles.

\section{Conclusions}

In the present work, the nucleation and crystallization of triple-crystalline immiscible blends based on PLA, PCL, and PBS exhibiting partial wetting morphology were investigated. Careful analysis of DSC and PLOM isothermal crystallization of each phase reveals several intriguing nucleation mechanisms.

When the highest melting polymer, PLA, is considered, crystallization can occur while the other phases remain in the molten state. Both molten PCL and PBS droplets manage to nucleate the PLA major phase via interfacial-assisted nucleation with a higher nucleation rate for the PCL interface. This peculiar observation can be explained on the base of a simple heterogeneous nucleation model. 
In sequential crystallization, the lower melting temperature component can nucleate on the surface of the previously crystallized one (with the higher melting temperature). This is the case for instance of PBS which nucleates on crystalline PLA in both binary and ternary blends. The possible existence of epitaxial relationships between the two polymers could be explored in the future.

The sequential nucleation or droplet-to-droplet nucleation phenomenon of the PBS droplets was attributed to the weak partial-wetting behavior of the 45/10/45 PLA/PBS/PCL ternary system which allow a higher mobility of the PBS droplets when all the system is in the melt state and results in the formation of thin continuous layers connecting the PBS droplets (complete wetting behavior). These layers are responsible for the transfer of the nucleation from droplet to droplet, in a sequential fashion.

The obtained results present the interplay between nucleation and crystallization on the one hand and the complex morphology of the immiscible ternary systems on the other and shows a promising pathway to control the crystallization and hence tune the final properties of multiphase polymer blends.

\section{Acknowledgement}

We would like to thank the financial support provided by the BIODEST project; this project has received funding from the European Union's Horizon 2020 research and innovation programme under the Marie Skłodowska-Curie grant agreement No 778092 . We would also like to acknowledge funding from MINECO through project MAT2017-83014C2-1-P and from the Basque Government through grant IT1309-19.

\section{References}

[1] Y.-H. Na, Y. He, X. Shuai, Y. Kikkawa, Y. Doi, Y. Inoue, Biomacromolecules 2002, 3 (6), 1179-1186.

[2] J. Urquijo, G. Guerrica-Echevarría, J. I. Eguiazábal, J. Appl. Polym. Sci. 2015, 132 (41).

[3] T. Gumede, A. Luyt, A. Muller, 2018.

[4] Z. Qiu, M. Komura, T. Ikehara, T. Nishi, Polymer 2003, 44 (25), 7749-7756.

[5] A. Ostafinska, I. Fortelny, M. Nevoralova, J. Hodan, J. Kredatusova, M. Slouf, RSC Adv. 2015, 5 (120), 98971-98982. 
[6] T. Yokohara, M. Yamaguchi, Eur. Polym. J. 2008, 44 (3), 677-685.

[7] M. E. Córdova, A. T. Lorenzo, A. J. Müller, L. Gani, S. Tencé-Girault, L. Leibler, Macromol. Chem. Phys. 2011, 212 (13), 1335-1350.

[8] P. B. Handbook Springer: 2014.

[9] M. Pracella, Handbook of Polymer Crystallization; Piorkowka, E., Rutledge, GC, Eds 2013, 287-307.

[10] N.-D. Tien, R. E. Prud'homme In Crystallization in Multiphase Polymer Systems; Elsevier: 2018; pp 181-212.

[11] H. Frensch, P. Harnischfeger, B.-J. Jungnickel ACS Publications: 1989;

[12] S. E. Fenni, D. Cavallo, A. J. Müller In Thermal Properties of Bio-based Polymers; Di Lorenzo, M. L.; Androsch, R., Eds; Springer International Publishing: Cham, 2019; pp 219256.

[13] F. Sakai, K. Nishikawa, Y. Inoue, K. Yazawa, Macromolecules 2009, 42 (21), 83358342 .

[14] W. Shi, F. Chen, Y. Zhang, C. C. Han, ACS Macro Lett. 2012, 1 (8), 1086-1089.

[15] H. Huo, C. Guo, J. Zhou, X. Zhao, Colloid. Polym. Sci. 2014, 292 (4), 971-983.

[16] P. Pan, G. Shan, Y. Bao, Ind. Eng. Chem. Res. 2014, 53 (8), 3148-3156.

[17] C. Yu, L. Han, J. Bao, G. Shan, Y. Bao, P. Pan, J. Phys. Chem. B 2016, 120 (32), 80468054.

[18] Y. Kong, Y. Ma, L. Lei, X. Wang, H. Wang, Polymers 2017, 9 (2), 42.

[19] Z. Bartczak, A. Galeski, N. Krasnikova, Polymer 1987, 28 (10), 1627-1634.

[20] W. Wenig, H. W. Fiedel, Die Makromolekulare Chemie 1991, 192 (2), 191-199.

[21] M. L. Di Lorenzo, S. Cimmino, C. Silvestre, J. Appl. Polym. Sci. 2001, 82 (2), 358-367.

[22] M. L. Di Lorenzo, P. Rubino, M. Cocca, J. Appl. Polym. Sci. 2014, 131 (12).

[23] M. L. Di Lorenzo, P. Rubino, M. Cocca, Eur. Polym. J. 2013, 49 (10), 3309-3317.

[24] S. Ravati, S. Poulin, K. Piyakis, B. D. Favis, Polymer 2014, 55 (23), 6110-6123.

[25] L. Wang, W. Ma, R. Gross, S. McCarthy, Polymer Degradation and Stability 1998, 59 (1-3), 161-168.

[26] F. Tuba, L. Oláh, P. Nagy, Engineering fracture mechanics 2011, 78 (17), 3123-3133.

[27] O. Persenaire, R. Quintana, Y. Lemmouchi, J. Sampson, S. Martin, L. Bonnaud, P. Dubois, Polym. Int. 2014, 63 (9), 1724-1731.

[28] S. Wachirahuttapong, C. Thongpin, N. Sombatsompop, Energy Procedia 2016, 89, 198206.

[29] S. Ravati, B. D. Favis, Polymer 2010, 51 (16), 3669-3684. 
[30] P. Le Corroller, B. D. Favis, Macromol. Chem. Phys. 2012, 213 (19), 2062-2074.

[31] J. Wang, B. H. Lessard, M. Maric, B. D. Favis, Polymer 2014, 55 (16), 3461-3467.

[32] A. M. Zolali, V. Heshmati, B. D. Favis, Macromolecules 2017, 50 (1), 264-274.

[33] J. Wang, A. Reyna-Valencia, B. D. Favis, Macromolecules 2016, 49 (14), 5115-5125.

[34] S. Ravati, B. D. Favis, Polymer 2010, 51 (20), 4547-4561.

[35] P. Le Corroller, B. D. Favis, Polymer 2011, 52 (17), 3827-3834.

[36] S. Ravati, B. D. Favis, Polymer 2013, 54 (25), 6739-6751.

[37] S. Ravati, C. Beaulieu, A. M. Zolali, B. D. Favis, AlChE J. 2014, 60 (8), 3005-3012.

[38] A. M. Zolali, B. D. Favis, J. Phys. Chem. B 2016, 120 (49), 12708-12719.

[39] A. M. Zolali, B. D. Favis, Polymer 2017, 114, 277-288.

[40] N. Nemirovski, A. Siegmann, M. Narkis, J. Macromol. Sci. Part B Phys. 1995, 34 (4), $459-475$.

[41] H. Guo, S. Packirisamy, N. Gvozdic, D. Meier, Polymer 1997, 38 (4), 785-794.

[42] C. Cerclé, B. D. Favis, Polymer 2012, 53 (20), 4338-4343.

[43] S. E. Fenni, J. Wang, N. Haddaoui, B. D. Favis, A. J. Müller, D. Cavallo, Macromolecules 2020, 53 (5), 1726-1735.

[44] A. T. Lorenzo, M. L. Arnal, J. Albuerne, A. J. Müller, Polymer testing 2007, 26 (2), 222231.

[45] V. Balsamo, N. Urdaneta, L. Pérez, P. Carrizales, V. Abetz, A. Müller, Eur. Polym. J. 2004, 40 (6), 1033-1049.

[46] S. Ravati, B. D. Favis, Polymer 2013, 54 (13), 3271-3281.

[47] S. Wu In Polymer interface and adhesion; CRC Press, Taylor \& Francic Group: New York, 1982; pp 1-28.

[48] S. E. Fenni, J. Wang, N. Haddaoui, B. D. Favis, A. J. Müller, D. Cavallo, Thermochimica Acta 2019, 677, 117-130.

[49] M. L. Di Lorenzo, R. Androsch, Polym. Int. 2019, 68 (3), 320-334.

[50] S. Fenni, O. Monticelli, L. Conzatti, R. Doufnoune, P. Stagnaro, N. Haddaoui, D. Cavallo, Express Polym. Lett. 2018, 12 (1), 58-70.

[51] M. Rizzuto, A. Mugica, M. Zubitur, D. Caretti, A. J. Müller, CrystEngComm 2016, 18 (11), 2014-2023.

[52] M. Rizzuto, L. Marinetti, D. Caretti, A. Mugica, M. Zubitur, A. J. Müller, CrystEngComm 2017, 19 (23), 3178-3191.

[53] A. M. Zolali, B. D. Favis, Soft matter 2017, 13 (15), 2844-2856.

[54] A. M. Zolali, B. D. Favis, Macromolecules 2018, 51 (10), 3572-3581. 
[55] X. Zhang, Z. Wang, M. Muthukumar, C. C. Han, Macromol. Rapid Commun. 2005, 26 (16), 1285-1288.

[56] X. Zhang, Z. Wang, X. Dong, D. Wang, C. C. Han, J. Chem. Phys. 2006, 125 (2), 024907.

[57] Y. Ma, L. Zha, W. Hu, G. Reiter, C. C. Han, Phys. Rev. E 2008, 77 (6), 061801.

[58] M. K. Mitra, M. Muthukumar, J. Chem. Phys. 2010, 132 (18), 184908.

[59] Q. Lv, D. Wu, H. Xie, S. Peng, Y. Chen, C. Xu, RSC Adv. 2016, 6 (44), 37721-37730.

[60] N. Virgilio, C. Marc-Aurele, B. D. Favis, Macromolecules 2009, 42 (9), 3405-3416.

[61] R. M. Michell, A. J. Müller, Prog. Polym. Sci. 2016, 54, 183-213.

\section{For Table of Contents Only}

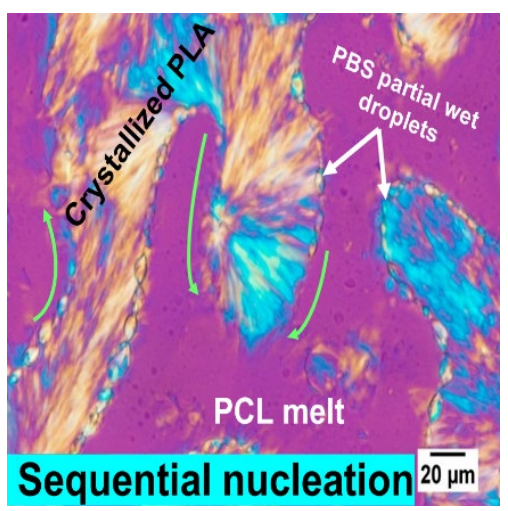

